\title{
Coherent manipulation of the Raman-induced gratings in atomic media
}

\author{
V. G. Arkhipkin ${ }^{1,2, *}$ and S. A. Myslivets ${ }^{1,3, \dagger}$ \\ ${ }^{1}$ L. V. Kirensky Institute of Physics, Krasnoyarsk 660036, Russia \\ ${ }^{2}$ Laboratory of Nonlinear Optics and Spectroscopy, Siberian Federal University, Krasnoyarsk 660079, Russia \\ ${ }^{3}$ Department of Photonics and Laser Technology, Siberian Federal University, Krasnoyarsk 660079, Russia
}

(Received 10 July 2015; published 8 January 2016)

\begin{abstract}
We consider dynamically controllable periodic structures (gratings), resulting from Raman interaction of a weak probe field with a standing-wave pump and a second control laser field in four-level atomic media of $N$ type. The gratings under study are induced due to periodic spatial modulation of the Raman gain in a standing pump field and fundamentally differ from the ones based on electromagnetically induced transparency. We show that spectral and transmission properties of these gratings can be controlled with the help of an additional weak field (control field) by varying its intensity or frequency. Small variations of the control field intensity can change the system from opaque to transparent and vice versa and this structure can operate as an all-optical transistor. Such a structure can also be used as a tunable nonlinear mirror with amplification.
\end{abstract}

DOI: 10.1103/PhysRevA.93.013810

\section{INTRODUCTION}

Nonlinear periodic optical media have important applications in controlling light propagation behavior, which has attracted a great deal of attention [1]. Spatially micromodulated materials such as photonic crystals (PCs) have been studied both theoretically and experimentally for more than two decades [2]. A large majority of PCs are formed by periodic modulation of the refractive index, i.e., modulation of the real part of the dielectric constant. The presence of absorption and (or) gain can radically change the nature of wave propagation in such materials, including the very notion of forbidden and allowed bands [3].

Quite a distinct approach to PCs uses optical resonant or quasiresonant nonlinearities that can be induced in a multilevel medium. Such nonlinearities can be exploited to generate spatially periodic structures required to devise an optically tunable photonic band gap (PBG). Unlike conventional PCs, here a periodic structure is created by external control light beams. Novel photonic structures could be created by optically inducing coherent nonlinearities based on electromagnetically induced transparency (EIT) [4] in a standing-wave coupling configuration [5]. When a standing-wave coupling field interacts with a three-level atomic system, the dispersion and absorption of a probe laser beam in an atomic medium is modulated spatially by the standing-wave coupling field. It has been proposed to induce spatially periodic quantum coherence for generation of a tunable PBG [6-10] and dynamic generation of stationary light pulses [11-13]. These structures are also referred to as electromagnetically induced absorption gratings (EIAG) [14] or electromagnetically induced gratings [15]. Many researchers have been focused on the study of EIAG [14-23], that are based on EIT, with their potential applications in mind. EIAG may be utilized for diffracting and switching a probe field, and probing the optical properties of materials.

\footnotetext{
*avg@iph.krasn.ru

†sam@iph.krasn.ru
}

Wave propagation in resonant optical media with an active-Raman-gain (ARG) core has also attracted considerable theoretical and experimental interest [24-29]. Unlike the EIT-based scheme, which is inherently absorptive, the central idea of the ARG scheme is that the probe (Raman) field operates in a stimulated Raman emission mode, and hence can eliminate signal attenuation and help realize stable subluminal (slow light) as well as superluminal (fast light) propagation of the probe wave even at room temperature [26-28]. Such systems could enable new practical applications of coherent processes. It has been shown that a gain-assisted giant Kerr effect [30-32] and superluminal solitons can be obtained using an ARG medium [33]. In papers [34-36] it was theoretically demonstrated how one could use an ARG medium together with a PC cavity to create all-optical switches and transistors.

Recently we proposed a concept of electromagnetically induced gratings in three-level atomic media, called Ramaninduced gratings (RIGs), on the basis of spatial modulation of the Raman nonlinearity in a standing-wave pump field [37]. These gratings are fundamentally different from the recent EIAG schemes, where the absorption is spatially modulated. In the present paper, we develop further this concept and propose a scheme to coherently control propagation of the probe (Raman) wave in a four-level atomic medium of $N$ type using an additional control field. Such an extension opens new possibilities for controlling transmission and spectral properties of the RIG. The transmission and reflection spectra can be controlled by varying the intensity or frequency of the control field. These schemes potentially could find application in optical transistors and amplifiers and be used for all-optical information processing.

\section{THEORETICAL MODEL AND BASIC EQUATIONS}

The four-level scheme of $N$-type configuration intended for coherent control of the Raman gain process is shown in Fig. 1. We consider an ensemble of lifetime broadening four-level atomic systems initially prepared in the ground state $|0\rangle$. The transitions $|0\rangle-|1\rangle,|1\rangle-|2\rangle$, and $|2\rangle-|3\rangle$ are electric dipole allowed while transitions $|0\rangle-|2\rangle$ and $|1\rangle-|3\rangle$ are electric dipole forbidden. The states $|0\rangle$ and $|1\rangle$ are coupled by a standing 


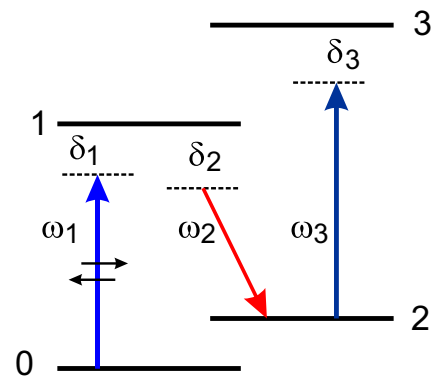

FIG. 1. Energy-level configuration and excitation scheme of a four-state ARG system of $N$ type, which interacts with a standing pump wave of frequency $\omega_{1}$, a weak traveling probe (Raman) field of frequency $\omega_{2}$, and a traveling control field of frequency $\omega_{3}$.

pump wave that is formed by two monochromatic counterpropagating pump fields $E_{p}=1 / 2\left\{E_{1}^{+} \exp \left[-i\left(\omega_{1} t-k_{1} z\right)\right]+\right.$ $\left.E_{1}^{-} \exp \left[-i\left(\omega_{1} t+k_{1} z\right)\right]\right\}$, where $E_{1}^{+}$and $E_{1}^{-}$are the amplitudes of the forward (FW) and backward (BW) wave, respectively, with frequency $\omega_{1}$ and wave vector $k_{1}$. For simplicity, we assume that these amplitudes are real. A traveling weak probe Raman wave $E_{s}=1 / 2 E_{2} \exp \left[-i\left(\omega_{2} t-k_{2} z\right)\right]$ and a control field $E_{c}=1 / 2 E_{3} \exp \left[-i\left(\omega_{3} t-k_{3} z\right)\right]$ also propagate along the $z$ direction and interact with the transitions $|1\rangle-|2\rangle$ and $|2\rangle-|3\rangle$, respectively. The pump field is detuned from state $|1\rangle$ with a large one-photon detuning so that single-photon absorption of the pump can be neglected. We will assume the probe field intensity to be much lower than the intensity of the pump field. The probe being weak, levels $|1\rangle$ and $|2\rangle$ remain empty and the space distribution of atoms remains homogeneous in the sample.

An off-resonant standing pump wave and the probe field induce spatially modulated coherence on the transition $|0\rangle-|2\rangle$ (off-diagonal element of the density matrix $\rho_{20}$ ), which leads to modulation of the Raman susceptibility. As a result, a medium with a spatially modulated Raman gain and nonlinear refractive index for the probe light is created. Therefore, the weak probe wave in such a medium propagates as in a one-dimensional periodic structure with the period $\Lambda=\lambda_{1} / 2$, where $\lambda_{1}$ is the wavelength of the pump field. The intensity of the pump is chosen so as not to exceed the stimulated Raman scattering threshold while being sufficiently strong to ensure a noticeable enhancement of the probe wave. The control field is applied to the transition $|2\rangle-|3\rangle$ which allows the Raman gain to be manipulated.

The Raman gain coefficient and the nonlinear (cross-Kerr) refractive index for the probe field are determined by the imaginary and real part of the macroscopic nonlinear Raman susceptibility $\chi_{n l}\left(\omega_{2}\right)$. Using the full density matrix equations and solving them exactly in the control field, to the second order in the pump field and the first order in the probe field, we find the susceptibility $\chi_{n l}\left(\omega_{2}\right)$, that depends on the pump and control fields [32]:

$$
\begin{aligned}
& \chi_{n l}\left(\omega_{2}\right)=\chi_{R}\left(\omega_{2}\right) N\left|E_{p}\right|^{2} \\
& \chi_{R}\left(\omega_{2}\right)=\frac{D_{02}\left(\Delta_{31} \Delta_{30}+\left|\Omega_{3}\right|^{2}\right)}{\Delta_{10}\left[\Delta_{20} \Delta_{30}-\left|\Omega_{3}\right|^{2}\right]\left[\Delta_{12}^{*} \Delta_{31}+\left|\Omega_{3}\right|^{2}\right]},
\end{aligned}
$$

where $E_{p}=E_{1}^{+} e^{i k_{1} z}+E_{1}^{-} e^{-i k_{1} z}, 2 \Omega_{3}=d_{23} E_{3} / \hbar$ is the Rabi frequency of the control field, $d_{i j}$ is the matrix dipole moment of the transition, $\hbar$ is the reduced Planck constant, $\Delta_{10}=\delta_{1}+i \gamma_{10}, \Delta_{12}=\delta_{2}+i \gamma_{12}, \Delta_{20}=\left(\delta_{1}-\delta_{2}\right)+i \gamma_{20}=$ $\delta_{20}+i \gamma_{20}, \Delta_{30}=\left(\delta_{1}-\delta_{2}+\delta_{3}\right)+i \gamma_{30}=\delta_{30}+i \gamma_{30}, \Delta_{31}=$ $\left(\delta_{3}-\delta_{2}\right)+i \gamma_{31}=\delta_{31}+i \gamma_{31}, \quad \delta_{j}=\omega_{m n}-\omega_{j} \quad$ are the frequency detunings, $\delta_{20}=\omega_{20}-\left(\omega_{1}-\omega_{2}\right), \delta_{30}=$ $\omega_{30}-\left(\omega_{1}-\omega_{2}+\omega_{3}\right), \delta_{31}=\omega_{31}-\left(\omega_{3}-\omega_{2}\right), \omega_{m n} \quad$ and $\gamma_{m n}$ are the frequency and half width of the respective transitions, $D_{02}=\left|d_{01}\right|^{2}\left|d_{12}\right|^{2} / 4 \hbar^{3}, N$ is the concentration of atoms. Hereinafter we assume that $\left|\delta_{1,2}\right| \gg \gamma_{10}, \gamma_{12}, \Omega_{1}$.

When the control field is weak $\left(\left|\Omega_{3}\right|^{2} \ll \gamma_{20} \gamma_{30} \mid\right)$, the susceptibility $\chi_{R}\left(\omega_{2}\right)$ can be expressed as

$$
\chi_{R}\left(\omega_{2}\right)=\frac{D_{02}}{\delta_{1} \delta_{2} \Delta_{20}}-\frac{D_{02}\left|\Omega_{3}\right|^{2}}{\delta_{1} \delta_{2} \Delta_{20}^{2} \Delta_{30}} .
$$

The first term in Eq. (2) is the ordinary Raman susceptibility in the absence of a control field $\Omega_{3}=0$ (the third order of perturbation theory) [38]. The second term is due to the control field and leads to modification of the Raman susceptibility (the fifth order of perturbation theory) [33].

The Raman gain and refractive index for the probe wave can be described by the dielectric function:

$$
\begin{aligned}
\varepsilon_{2}(z) & =1+4 \pi N \chi_{l}\left(\omega_{2}\right)+4 \pi N \chi_{n l}\left(\omega_{2}\right) \\
& =\varepsilon_{2 l}+\Delta \varepsilon\left[1+p \cos \left(2 k_{1} z\right)\right] .
\end{aligned}
$$

Here $\varepsilon_{2 l}=1+4 \pi N \chi_{l}\left(\omega_{2}\right), \chi_{l}\left(\omega_{2}\right)$ is the off-resonant microscopic linear susceptibility of the probe field, $\Delta \varepsilon=4 \pi N \chi_{R}\left(\omega_{2}\right)\left|E_{p}\right|^{2},\left|E_{p}\right|^{2}=\left|E_{1}^{+} e^{i k_{1} z}+E_{1}^{-} e^{-i k_{1} z}\right|^{2}=$ $\left(E_{1}^{2+}+E_{1}^{2-}\right)\left[1+p \cos \left(2 k_{1} z\right)\right], p=2 E_{1}^{+} E_{1}^{-} /\left(E_{1}^{2+}+E_{1}^{2-}\right)$. Clearly, the modulation depth is maximal when $p=1$, that is, $E_{1}^{+}=E_{1}^{-}=E_{1}$ (the ideal or perfect standing wave). In this case $\left|E_{p}\right|^{2}$ varies periodically from zero to $4 E_{1}^{2}$. The pump intensity vanishes at the nodal positions around which the atoms do not amplify probe radiation because Raman gain is zero. It should be noted that $|\Delta \varepsilon| \ll 1$, which corresponds to a shallow depth of modulation. When $\delta_{20} \neq 0$, a hybrid RIG is induced in the medium: a gain grating and a refraction grating. The former is an amplitude grating, and the latter is a phase grating.

Let us consider normal incidence of a weak probe wave on a one-dimensional periodic medium with the permittivity (3). A sample of finite length $L$ contains a very large number of periods $\Lambda$. The wave equation for the electric-field strength $E_{2}(z)$ in a medium with a spatially modulated dielectric constant has the form [39]

$$
\frac{d^{2} E_{2}}{d z^{2}}+k_{20}^{2} \varepsilon_{2}(z) E_{2}=0 .
$$

Considering Eq. (3), Eq. (4) can be reduced to

$$
\frac{d^{2} E_{2}}{d z^{2}}+k_{2}^{2}\left\{1+\mu\left[1+p \cos \left(2 k_{1} z\right)\right]\right\} E_{2}=0,
$$

where $k_{20}=\omega_{2} / c, k_{2}^{2}=k_{20}^{2} \varepsilon_{2 l}, \mu=\Delta \varepsilon / \varepsilon_{2 l}$, and $c$ is the velocity of light in vacuum.

By using the method of coupled waves [39], solution of Eq. (5) can be represented as a superposition of two waves 
propagating in opposite directions:

$$
E_{2}(z)=A(z) e^{i k_{2} z}+B(z) e^{-i k_{2} z},
$$

where $A(z)$ and $B(z)$ are the amplitudes of the FW and BW wave, respectively. Substituting Eq. (6) into Eq. (5) and using slowly varying amplitudes approximation $k_{2} d E_{2} / d z \gg$ $d^{2} E_{2} / d z^{2}$ we obtain a system of two coupled equations for $A(z)$ and $B(z)$ :

$$
\begin{aligned}
& \frac{d A}{d z}=i \alpha A+i \sigma B e^{i 2 \Delta k z}, \\
& \frac{d B}{d z}=-i \alpha B-i \sigma A e^{-i 2 \Delta k z},
\end{aligned}
$$

where $\alpha=k_{2} \mu / 2, \sigma=k_{2} p \mu / 4, \Delta k=k_{1}-k_{2}$. The parameter $\sigma$ determines the coupling strength of two counterpropagating fields.

Since $|\Delta \varepsilon| \ll 1$, we can neglect the Fresnel reflection from the layer boundaries and take into account only the volume reflection [40]. Then the boundary conditions can be written as $A(0)=A_{0}, B(L)=0$, where $A_{0}$ is the incident probe wave amplitude. By using these boundary conditions we find the solution to Eqs. (7) [37]:

$$
\begin{aligned}
& A(z)=A_{0}[s \cos s(L-z)+i(\Delta k-\alpha) \sin s(L-z)] / D, \\
& B(z)=A_{0}[\sigma \sin s(L-z)] / D,
\end{aligned}
$$

where $\quad D=s \cos (s L)+i(\Delta k-\alpha) \sin (s L)$ and $s=\sqrt{(\Delta k-\alpha)^{2}-\sigma^{2}}$.

The transmission $T$ and reflection $R$ coefficients are given as

$$
\begin{aligned}
& T=\frac{|A(L)|^{2}}{\left|A_{0}\right|^{2}}=\left|\frac{s}{s \cos (s L)+i(\Delta k-\alpha) \sin (s L)}\right|^{2}, \\
& R=\frac{|B(0)|^{2}}{\left|A_{0}\right|^{2}}=\left|\frac{\sigma \sin s L}{s \cos (s L)+i(\Delta k-\alpha) \sin (s L)}\right|^{2} .
\end{aligned}
$$

Equations (9) are similar to those for distributed-feedback lasers and backward parametric amplification in ordinary media and metamaterials [38,41]. It is obvious that the reflectivity and transmittance undergo resonance as functions of the slab thickness $L$ and of the pump or control field intensity, when the denominator in Eqs. (9) tends to zero. They are called "geometrical resonance" [42]. If $L=$ const., then these resonances manifest themselves as field resonances. Such behavior of $T$ and $R$ is in a drastic contrast with the Raman gain in a homogeneous medium, where the probe would grow exponentially and $R=0$ [43].

\section{NUMERICAL RESULTS AND DISCUSSION}

Spectral properties of the RIG in the absence of a control field $\left(\Omega_{3}=0\right)$ are considered in our paper [37], assuming that the pump field is a perfect standing wave $\left(E_{1}^{+}=E_{1}^{-}=E_{1}\right)$. In Fig. 2, the transmittance $T$ and the reflectivity $R$ for the probe are plotted as functions of the Raman detuning $\delta_{20}$ and pump Rabi frequency $\Omega_{1}=d_{01} E_{1} / \hbar$. It can be seen that the transmission and reflection spectra strongly depend on the pump intensity. The transmitted and reflected light can be simultaneously amplified in some frequency range. Therefore, transmittance and reflectivity can be interpreted as
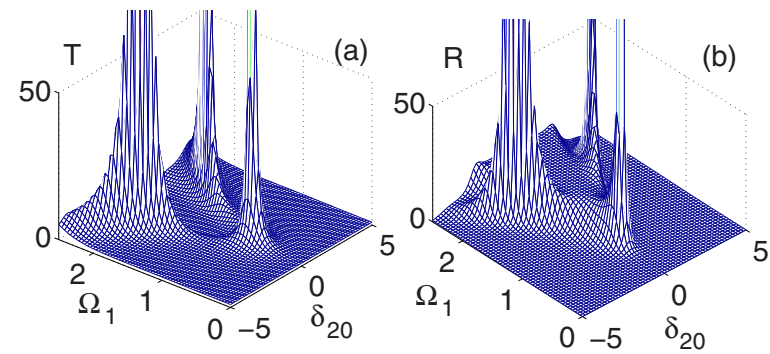

FIG. 2. Transmission (a) and reflection (b) vs the Raman detuning $\delta_{20}$ (in units $\gamma_{20}$ ) and pump Rabi frequency $\Omega_{1}$ (in units $\gamma_{10}$ ) under $\delta_{1}=30 \gamma_{10}$.

a transmission gain and a reflection gain, respectively. Near the Raman resonance the transmission $T \rightarrow 0$ with increasing $\Omega_{1}$ and the transparent medium becomes opaque. Away from the Raman resonance $T \rightarrow 1$ and $R \rightarrow 0$. Note that this structure can also be considered as a nonlinear tunable mirror with the reflectivity $R>1$ [Fig. 2(b)].

The peaks in Fig. 2 correspond to the spectral and geometrical resonances. Geometric resonance corresponds to the maximal transmission (reflection) at the optimal value $\Omega_{1}$, depending on the length of the sample. Transmission and reflection outside the region of the geometrical resonance are minimal. We note that the transmission and reflection are determined not only (and not so much) by the Raman susceptibility but also and to a greater extent by resonant properties of the structure, depending on the intensity of the pump field. Such an unusual behavior of the resonance transmission and reflection as a function of the intensity of the pump field and the thickness of the sample is due to the fact that the probe wave propagates in both forward and backward directions. A similar behavior is typical of distributed-feedback lasers and is equivalent to greatly extending the nonlinear coupling length [38].

We emphasize that the transmission and reflection spectra are formed by joint action of the gain grating and the refraction grating. The latter occurs when $\delta_{20} \neq 0$. Therefore RIG is a hybrid grating.

In the paper we consider an ARG scheme (Fig. 1) when the control field is switched on $\left(\Omega_{3} \neq 0\right)$. In this case, the nonlinear Raman susceptibility is determined by Eqs. (1). The nonlinear susceptibility $\chi_{n l}$ depends on the intensity of the control field. By varying the intensity it is possible to control $\operatorname{Im} \chi_{n l}$ and $\operatorname{Re} \chi_{n l}$ and thereby control the spectral properties of the RIG. For numerical simulations we use parameters corresponding to the $D_{1}$ line of $\mathrm{Na}$ atoms, and the levels $|0\rangle$ and $|2\rangle$ are long-lived hyperfine sublevels of the electronic ground state $3 S_{1 / 2}$. The atomic parameters are $\gamma_{10}=2 \pi \times 10 \mathrm{MHz}$, $\gamma_{20}=\gamma_{10} / 100, \gamma_{10}=\gamma_{30}, \delta_{1}=30 \gamma_{10}, N=10^{12} \mathrm{~cm}^{-3}$, and the sample length is $L=10^{3} \Lambda \simeq 0.6 \mathrm{~mm}$.

In Fig. 3 we have plotted the dependences of $\operatorname{Im}\left[\chi_{n l}\left(\omega_{2}\right)\right]$ characterizing the Raman gain spectrum and $\operatorname{Re}\left[\chi_{n l}\left(\omega_{2}\right)\right]$ characterizing the refractive spectrum as functions of the Raman detuning $\delta_{20}$ with different values of $\Omega_{3}$. For small Rabi frequencies of the control field, the gain profile of the probe field exhibits a maximum near the resonance $\left(\delta_{20}=0\right)$. It is essential that the imaginary part of $R$ is negative near the Raman resonance-the negative absorption or amplification; 


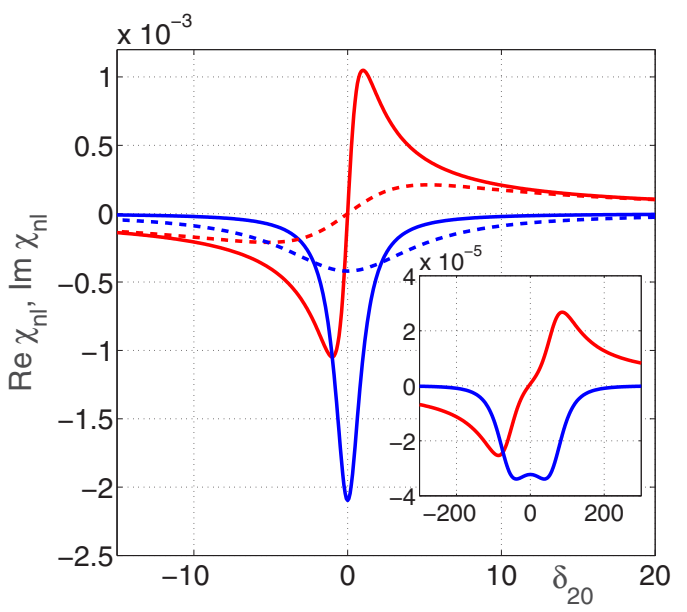

FIG. 3. Variation of $\operatorname{Im} \chi_{n l}$ (blue) and $\operatorname{Re} \chi_{n l}$ (red) with Raman detuning $\delta_{20}$ for various Rabi frequencies $\Omega_{3}: \Omega_{3}=0$, solid curves; $\Omega_{3}=0.2 \gamma_{10}$, dashed curves. Insets: The same dependences for Raman susceptibility under $\Omega_{3}=0.8 \gamma_{10} . \Omega_{1}=1.5 \gamma_{10}$. The other parameters are given in the text.

i.e., the probe wave is enhanced due to the energy transfer from the pump radiation to the probe field. The imaginary and real parts of the susceptibility decrease with increasing $\Omega_{3}$. When $\Omega_{3}$ exceeds some critical value $\Omega_{3}^{\mathrm{cr}}$, the gain peak splits into a doublet, i.e., a dip in the line center of $\operatorname{Im}\left[\chi_{n l}\left(\omega_{2}\right)\right]$ is formed $[32,33,36]$. The width and depth of the dip increase with $\Omega_{3}$. We stress that the physical reason for the appearance of the gain doublet lies in the quantum interference effect induced by the control field [32].

Let us show that using the control field with $\Omega_{3}<\Omega_{3}^{\text {cr }}$, makes it possible to effectively control the spectral properties of the RIG. The control field intensity may be much smaller than the intensity of the pump field. First, we assume that a standing-wave pump is perfect, i.e., $E_{1}^{+}=E_{1}^{-}=E_{1}$. Figure 4 shows transmission (a) and reflection (b) of the probe field as a function of the Raman detuning $\delta_{20}$ for different Rabi frequencies of the control field $\Omega_{3}$. Initially, when $\Omega_{3}=0$, the pump intensity is chosen such that the transmission is close to zero at $\delta_{20}=0$. As can be seen from Fig. 4, the transmission and reflection spectra are sensitive to the strength of the control field. Transmission and reflection dependences on the Rabi frequency of the control field have a resonance character
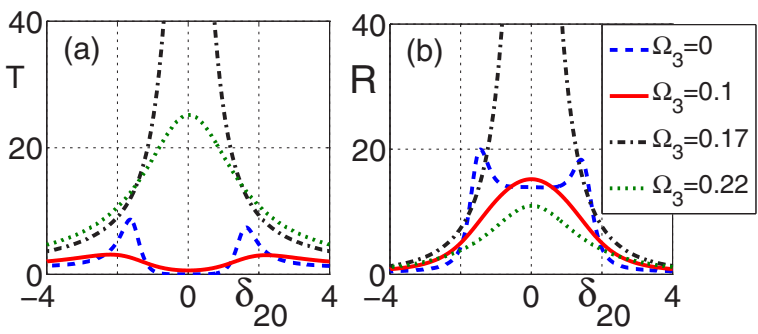

FIG. 4. Transmission $T$ (a) and reflection $R$ (b) as a function of the Raman detunings $\delta_{20}$ (in units $\gamma_{20}$ ) for different Rabi frequencies of the control field $\Omega_{3}$ (in units $\gamma_{10}$ ) under $\Omega_{1}=1.5 \gamma_{10}$, $\delta_{3}=0$.
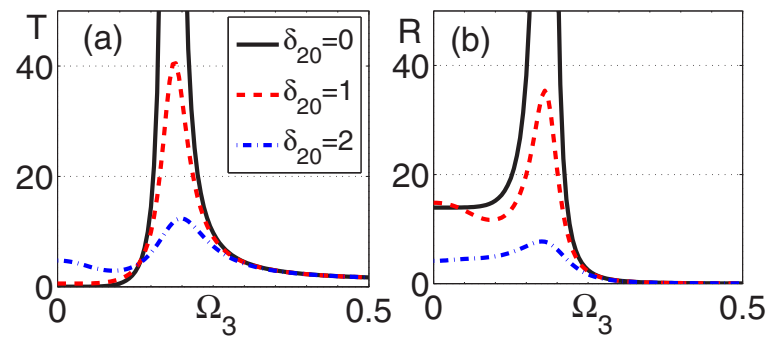

FIG. 5. Transmission $T$ and reflection $R$ as a function of the Rabi frequency of the control field $\Omega_{3}$ (in units $\gamma_{10}$ ) for different Raman detunings $\delta_{20}$ (in units $\gamma_{20}$ ) under $\Omega_{1}=1.5 \gamma_{10}$, $\delta_{3}=0$.

(Fig. 5). The magnitude of the resonance peaks depends on the Raman detuning.

If under $\Omega_{3}=0$ the transmission (reflection) is maximum, applying the control field reduces it (Fig. 6). As $\Omega_{3}$ grows, $T \rightarrow 1$. The transmission and reflection can also be changed by varying the frequency of the control field as seen from Fig. 7.

Note that small variations of the control field intensity can change the system from opaque to transparent (with amplification) and vice versa (see Figs. 4 and 5). Therefore the weak optical control field can serve as a gate field of the transistor to effectively control propagation of the probe, i.e., this structure can operate as an all-optical transistor. It can also be used as a controlled nonlinear mirror with amplification of the reflected signal.

Figure 8 demonstrates a typical behavior of $T$ and $R$ as functions of the Raman detuning and the Rabi frequency of the control field for different values of the Rabi frequency of the pump field. The figures shows that for certain values of $\Omega_{3}$ the resonance peaks appear in the transmission and reflection. Their position depends on the intensity of the pump field. Note that the field intensity required for emergence of resonances depends on the thickness of the sample $L$. The intensity reduces as $L$ grows. Therefore the resonances may be called geometrical resonances.

Generally, $E_{1}^{+} \neq E_{1}^{-}$and the standing wave is not perfect. In this case the pump intensity does not vanish anywhere and the nodes become quasinodes where Raman amplification takes place. Formulas for $\Delta \varepsilon$ and $p$ in Eq. (3) are conveniently represented as $\Delta \varepsilon=4 \pi \chi_{R} N E_{1}^{+}\left(1+a^{2}\right)$ and $p=2 a /\left(1+a^{2}\right)$,
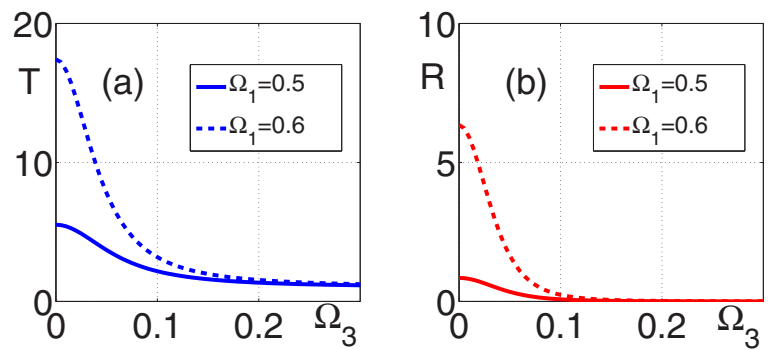

FIG. 6. Transmission $T$ and reflection $R$ as a function of the control Rabi frequency $\Omega_{3}$ (in units $\gamma_{10}$ ) for different pump Rabi frequencies $\Omega_{1}$ (in units $\gamma_{10}$ ) under the Raman resonance $\delta_{20}=0$. 

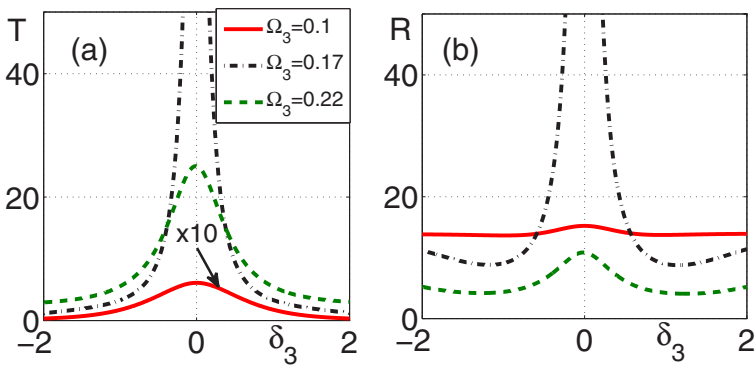

FIG. 7. Transmission $T$ (a) and reflection $R$ (b) as a function of control frequency detuning $\delta_{3}$ (in units $\gamma_{10}$ ) for different Rabi frequencies $\Omega_{3}$ (in units $\gamma_{10}$ ) under $\Omega_{1}=1.5 \gamma_{10}$ and $\delta_{20}=0$.

where $a=E_{1}^{-} / E_{1}^{+}=\Omega_{1}^{-} / \Omega_{1}^{+}\left(\Omega_{1}^{ \pm}=d_{01} E_{1}^{ \pm} / \hbar\right.$ are the Rabi frequencies for $\mathrm{FW}$ and $\mathrm{BW}$ waves).

Figure 9 shows the transmission and reflection spectra for different values of the parameter $a$ under $\Omega_{3}=0$. Here, the solid curves correspond to the ideal pump standing wave $(a=1)$. It is seen that with the decrease of parameter $a$ the peaks and the depth of the dip are reduced. This is caused by the fact that the modulation depth of the grating (parameter p) decreases. In Fig. 10 we have plotted transmission and reflection dependences as a function of the Rabi frequency
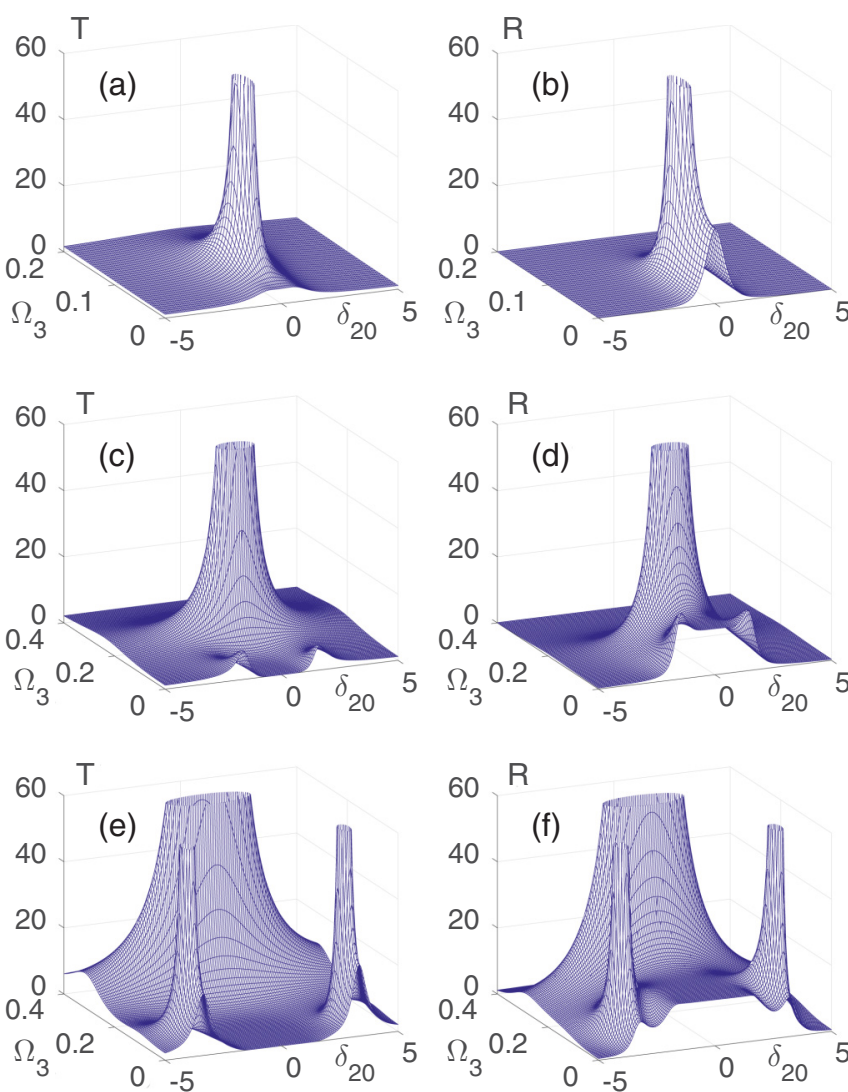

FIG. 8. The transmission $T$ (a,c,e) and reflection $R$ (b,d,f) spectra as a function of the Raman detuning $\delta_{20}$ (in units $\gamma_{20}$ ) and the Rabi frequency $\Omega_{3}$ in units $\gamma_{10}$ for various Rabi frequencies of the control field $\Omega_{1}$. (a,b) $\Omega_{1}=0.9 \gamma_{10}$. (c,d) $\Omega_{1}=1.5 \gamma_{10}$. (e,f) $\Omega_{1}=2.2 \gamma_{10}$. $\delta_{3}=0$. The other parameters are given in the text.
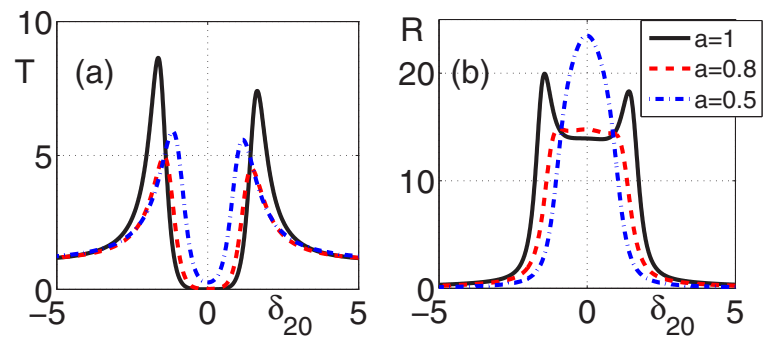

FIG. 9. The transmission $T$ (a) and reflection $R$ (b) spectra vs Raman detuning $\delta_{20}$ (in units $\gamma_{20}$ ) for various values of the parameter a. $\Omega_{1}^{+}=1.5 \gamma_{10}$.

of the control field. They are the same as for $a=1$, but the resonance peaks are shifted toward lower control fields. Thus, the transmission and reflection are sensitive to the value of $a$, but not critical.

\section{CONCLUSIONS}

In conclusion, we have carried out a detailed analysis of the probe field propagation in four-level atomic media under Raman interaction with a standing-wave pump field and an additional control laser radiation. The probe field experiences periodic variations of the Raman gain and the refractive index that arise due to the spatial modulation of the permittivity with a period $\Lambda=\lambda_{1} / 2$. Therefore this structure acts as a hybrid grating (a gain and refraction grating) and can dramatically change the spectral and transmission properties of the medium.

An additional weak control field opens new possibilities of controlling the spectral and propagation properties of RIG. It is shown that the transmission and reflection as a function of the intensity of the control field have a resonance character. It is also shown that a transparent medium with periodic spatial modulation of the Raman gain can be opaque near the Raman resonance. Small variations of resonant control field intensity can change the system from opaque to transparent and vice versa. Such controllable transmission is well suited to study alloptical switching at low control field intensities and create an all-optical transistor. At the same time, this structure represents a nonlinear controllable mirror with $R>1$. Also note that a geometrical resonance where gain may exceed a threshold of stimulated Raman scattering can provide a mirrorless Raman laser.
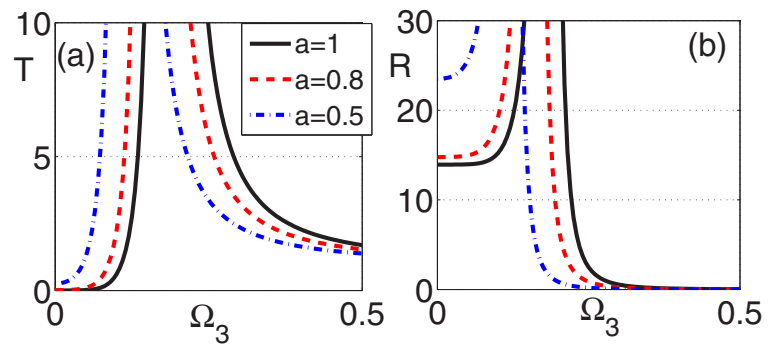

FIG. 10. Transmission $T$ (a) and reflection $R$ (b) as a function of the control field Rabi frequency $\Omega_{3}$ (in units $\gamma_{10}$ ) for different values of the parameter $a . \Omega_{1}^{+}=1.5 \gamma_{10}, \delta_{20}=\delta_{3}=0$. 
The control and pump field intensity depend on a number of factors (detuning from the resonance, the Raman resonance width, and length of the medium) and can be $10-100 \mu \mathrm{W} / \mathrm{cm}^{2}$ for the control field and $10-100 \mathrm{~mW} / \mathrm{cm}^{2}$ for the pump. The results obtained are in good agreement with the exact calculations based on the recurrence-relations technique [44]. For experimental realization room-temperature and ultracold atoms or ions as well as molecular gases can be used. These experiments are similar to [14]. Hollow-core photonic crystal fibers filled with atoms can be used to reduce the pump and control field intensity requirements. Additionally, it would be interesting to extend our scheme to diamond with nitrogen vacancy color centers or crystals of Pr doped $\mathrm{Y}_{2} \mathrm{SiO}_{5}$.

\section{ACKNOWLEDGMENT}

This work was supported by the Russian Foundation for Basic Research through Grant No. 15-02-03959.
[1] Nonlinearities in Periodic Structures and Metamaterials, edited by C. Denz, S. Flach, and Y. S. Kivshar, Springer Series in Optical Sciences Vol. 150 (Springer-Verlag, New York, 2009).

[2] J. D. Joannopoulos, S. G. Johnson, J. N. Winn, and R. D. Meade, Photonic Crystals: Molding the Flow of Light, 2nd ed. (Princeton University, Princeton, NJ, 2008).

[3] N. N. Rozanov, S. V. Fedorov, R. S. Savel'ev, A. A. Sukhorukov, and Y. S. Kivshar, JETP 114, 782 (2012).

[4] M. Fleischhauer, A. Imamoglu, and J. P. Marangos, Rev. Mod. Phys. 77, 633 (2005).

[5] J.-H. Wu, A. Raczyński, J. Zaremba, S. Zielińska-Kaniasty, M. Artoni, and G. La Rocca, J. Mod. Opt. 56, 768 (2009).

[6] A. André and M. D. Lukin, Phys. Rev. Lett. 89, 143602 (2002).

[7] X. M. Su and B. S. Ham, Phys. Rev. A 71, 013821 (2005).

[8] M. Artoni and G. C. La Rocca, Phys. Rev. Lett. 96, 073905 (2006).

[9] S.-q. Kuang, R.-g. Wan, P. Du, Y. Jiang, and J.-y. Gao, Opt. Express 16, 15455 (2008).

[10] J.-H. Wu, G. C. La Rocca, and M. Artoni, Phys. Rev. B 77, 113106 (2008).

[11] M. Bajcsy, A. S. Zibrov, and M. D. Lukin, Nature (London) 426, 638 (2003).

[12] K. R. Hansen and K. Molmer, Phys. Rev. A 75, 053802 (2007).

[13] S. A. Moiseev, A. I. Sidorova, and B. S. Ham, Phys. Rev. A 89, 043802 (2014).

[14] A. W. Brown and M. Xiao, Opt. Lett. 30, 699 (2005).

[15] H. Y. Ling, Y.-Q. Li, and M. Xiao, Phys. Rev. A 57, 1338 (1998).

[16] M. Mitsunaga and N. Imoto, Phys. Rev. A 59, 4773 (1999).

[17] P.-W. Zhai, X.-M. Su, and J.-Y. Gao, Phys. Lett. A 289, 27 (2001).

[18] G. C. Cardoso and J. W. R. Tabosa, Phys. Rev. A 65, 033803 (2002).

[19] B. K. Dutta and P. K. Mahapatra, J. Phys. B 39, 1145 (2006).

[20] I.-H. Bae, H. S. Moon, M.-K. Kim, L. Lee, and J. B. Kim, Appl. Opt. 47, 4849 (2008).

[21] L. E. E. de Araujo, Opt. Lett. 35, 977 (2010).
[22] Z.-H. Xiao, S. G. Shin, and K. Kim, J. Phys. B 43, 161004 (2010).

[23] J. Wen, Y.-H. Zhai, S. Du, and M. Xiao, Phys. Rev. A 82, 043814 (2010).

[24] L. J. Wang, A. Kuzmich, and A. Dogariu, Nature (London) 406, 277 (2000).

[25] A. Dogariu, A. Kuzmich, and L. J. Wang, Phys. Rev. A 63, 053806 (2001).

[26] M. G. Payne and L. Deng, Phys. Rev. A 64, 031802 (2001).

[27] K. J. Jiang, L. Deng, and M. G. Payne, Phys. Rev. A 74, 041803 (2006).

[28] K. J. Jiang, L. Deng, and M. G. Payne, Phys. Rev. A 76, 033819 (2007).

[29] F. L. Kien and K. Hakuta, Can. J. Phys. 78, 543 (2000).

[30] L. Deng and M. G. Payne, Phys. Rev. Lett. 98, 253902 (2007).

[31] K. J. Jiang, L. Deng, E. W. Hagley, and M. G. Payne, Phys. Rev. A 77, 045804 (2008).

[32] G. S. Agarwal and S. Dasgupta, Phys. Rev. A 70, 023802 (2004).

[33] C. Hang and G. Huang, Opt. Express 18, 2952 (2010).

[34] V. G. Arkhipkin and S. A. Myslivets, Phys. Rev. A 80, 061802 (2009).

[35] V. G. Arkhipkin and S. A. Myslivets, JETP 111, 898 (2010).

[36] V. G. Arkhipkin and S. A. Myslivets, Phys. Rev. A 88, 033847 (2013).

[37] V. G. Arkhipkin and S. A. Myslivets, Opt. Lett. 39, 3223 (2014).

[38] A. Yariv, Quantum Electronics, 3rd ed. (Wiley, New York, 1989).

[39] S. Rautian, Opt. Spectrosc. 104, 112 (2008).

[40] S. Y. Karpov and S. N. Stolyarov, Phys.-Usp. 36, 1 (1993).

[41] A. K. Popov, S. A. Myslivets, and V. M. Shalaev, Opt. Lett. 34, 1165 (2009).

[42] A. K. Popov and V. M. Shalaev, Proc. SPIE 8093, 809306 (2011).

[43] R. W. Boyd, Nonlinear Optics (Academic, London, 1992).

[44] V. G. Arkhipkin and S. A. Myslivets, Quantum Electron. 39, 157 (2009). 